\title{
Micromagnetic Simulations of Submicron Cobalt Dots
}

G.J. Parker, C. Cerjan

This article was submitted to the $44^{\text {th }}$ Annual Conference on Magnetism and Magnetic Materials, San Jose, CA, November 15-18, 1999

\section{August 30, 1999}

U.S. Department of Energy

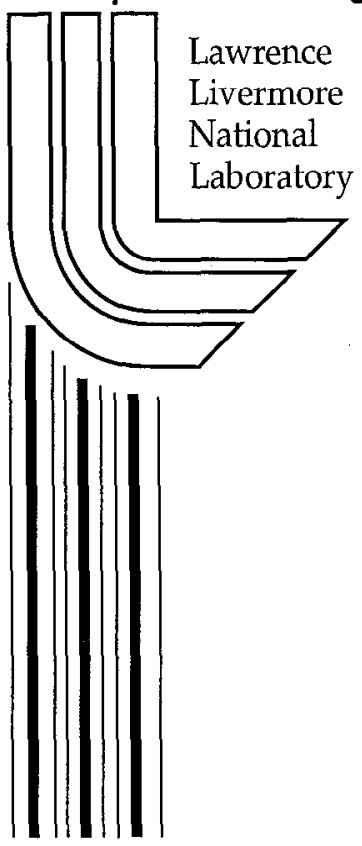





\section{DISCLAIMER}

This document was prepared as an account of work sponsored by an agency of the United States Government. Neither the United States Government nor the University of California nor any of their employees, makes any warranty, express or implied, or assumes any legal liability or responsibility for the accuracy, completeness, or usefulness of any information, apparatus, product, or process disclosed, or represents that its use would not infringe privately owned rights. Reference herein to any specific commercial product, process, or service by trade name, trademark, manufacturer, or otherwise, does not necessarily constitute or imply its endorsement, recommendation, or favoring by the United States Government or the University of California. The views and opinions of authors expressed herein do not necessarily state or reflect those of the United States Government or the University of California, and shall not be used for advertising or product endorsement purposes.

This is a preprint of a paper intended for publication in a journal or proceedings. Since changes may be made before publication, this preprint is made available with the understanding that it will not be cited or reproduced without the permission of the author.

This report has been reproduced

directly from the best available copy.

Available to DOE and DOE contractors from the

Office of Scientific and Technical Information

P.O. Box 62, Oak Ridge, TN 37831

Prices available from (423) 576-8401

http:/ / apollo.osti.gov/bridge/

Available to the public from the

National 'lechnical Information Service

U.S. Department of Commerce

5285 Port Royal Rd., Springfield, VA 22161

http://www.ntis.gov/

OR

Lawrence Livermore National Laboratory

Technical Information Department's Digital Library

http://www.llnl.gov/tid/Library.html 

MICROMAGNETIC SIMULATIONS OF SUBMICRON COBALT DOTS

\author{
G.J. Parker and C. Cerjan
}

Lawrence Livermore National Laboratory,7000 East Avenue, L-395, Livermore, CA 94550.

\begin{abstract}
Numerical simulations of submicron Co extruded elliptical dots were performed to illustrate the relative importance of different physical parameters on the switching behavior in the easy direction. Shape, size, magnetic moment magnitude and crystalline anistropy, both magnitude and distribution, were varied. The simulation includes calculation of the magnetostatic, exchange and crystalline anistropy fields on a structured mesh using finite difference techniques. The smooth boundary of the dots are accurately represented by use of the Embedded Curve Boundary method.
\end{abstract}

PACS: $75.40 \mathrm{Mg}, 75.50 .-\mathrm{d}, 85.70 .-\mathrm{w}$

Keywords: Micromagnetic simulation, Co dots, magnetic reversal, magnetic vortex.

\title{
Corresponding Author:
}

Charles Cerjan

Lawrence Livermore National Laboratory

7000 East Avenue, L-395

Livermore, CA 94551

(925) 423-8032, (925) 423-1488 (FAX)

cerjan1@llnl.gov 


\section{Introduction}

Mircomagnetics is an approximation [1] useful for modeling the behavior of small scale (anti)ferromagnetic materials. This continuum approximation efficiently and accurately models the response of magnetic materials for important commercial and industrial applications, including but not limited to read/write heads for magnetic storage, properties and performance of magnetic storage media, and non-volatile magnetic random access memory (MRAM). Furthermore, such simulations not only offer detailed information on the interactions of various physical mechanisms affecting their response, but also holds the potential of increasing the fundamental understanding of micromagnetic materials.

In this paper, we perform a series of simulations using a previously reported algorithm [2]. Briefly, this algorithm uses a structured (orthogonal Cartesian 3-D) mesh to calculate the magnetostatic and exchange fields by finite differences. Smooth curved boundaries are included in the algorithm by approximating the curved boundary by sub-mesh piecewise linear line segments which, when combined with the basic boundary conditions of the magnetic field, give rise to modified finite differencing equations as described in Ref. 2. Once the appropriate fields are calculated, the Landau-Lillshitz-Gilbert equation is semi-implicitly advanced in time. The two calculations (field and magnetic moment relaxation) are repeated until user specified criteria are reached. The algorithm was shown to be fast and robust and agree well with other simulations' results. [2]

Here we are concerned about the magnetic responsc of submicron polycrystalline Cobalt elliptical dots of varying thickness $(16-40 \mathrm{~nm})$ along the easy direction. The dots have major and minor axes of $a=450 \mathrm{~nm}$ and $b=250 \mathrm{~nm}$, respectively. The magnetic moment, $M_{s}$, derived from experiment ranged from 1160 to $1450 \mathrm{emu} / \mathrm{cm}^{3}$. An exchange coupling coefficient of $A=1.6 \times 10^{-6} \mathrm{erg} / \mathrm{cm}^{2}$ along with the crystalline anistropy coefficient of $K=7.5 \times 10^{5} \mathrm{erg} / \mathrm{cm}^{3}$ and a nominal grain size of $6 \mathrm{~nm}$ were also taken from experimental results. Detailed experimental procedures and results can be found in [3].

As shown previously, good agreement between simulation and experiments were obtained inde-

pendent of the 2-D or 3-D [2,4] random distribution of the crystalline anistropy, $\hat{k}$, for the magnetic 
response in the easy direction. Experimentally, the switching of the dots were seen to originate from a nucleation of a single or double vortex in the magnetic configuration at small values of an uniformly applied external field. Larger fields were then needed to completely switch the magnetic configuration. Simulation results predicted both the coercivity and the required external field needed to completely switch the magnetic configuration. However, the simulation predicted that single vortex formation occurs $50 \%$ of the time instead of the much larger experimentally observed 97\%. Furthermore, unlike the experimental result of a gradual nucleation of the single vortex state at low applied fields, simulations predict a sudden onset of the vortex state near the coercivity field, $H_{c}$.

In order to illustrate the effects of different physical parameters on the magnetic response, we have varied numerous parameters in the simulations to determine the relative importance of each. The physical parameters varied include: shape and size of the dots, the magnetic moment $M_{s}$, the magnitude of the crystalline anistropy $K$, the direction of the crystalline anistropy $\hat{k}$ and the grain size and coupling. The exchange field coefficient $A$ was not varied and was fixed to $1.6 \times 10^{6}$ $\mathrm{erg} / \mathrm{cm}^{2}$.

In all the simulations reported here, the ellipse was centered on the uniformly discretized mesh. The easy direction (i.e. the major axis) was placed along the $x$-axis while the minor axis was along $y$. The mesh spacing in the $z$ direction was adjusted so that 10 computational cells resided in the thickness of the dot. The $x$ and $y$ mesh spacing was fixed at $6 \mathrm{~nm}$. Around this uniformly discretized mesh, geometrically increasing mesh size of 20 cells in each of the 6 directions were placed so that the total length was much larger than any of the dot length scales. This was required to have a Dirichlet boundary condition of zero at 'infinity' for the magnetostatic potential calculation. An external uniform applied field along the $x$-axis was used to invoke the magnetic response of the dots. All dots were initially given a magnetic moment configuration of a single vortex.

The organization of this presentation is straightforward. In Section II, the effects of shape/size of the dots and the magnitude of the magnetic moment on the magnetic response is first performed. Section III shows the effect of uniform distributions of the crystalline anistropy on the magnetic: 
response. Finally, Section IV shows some effects of different random orientations of the crystalline anistropy.

\section{Shape, Size and Magnetic Moment Magnitude}

In this section, a series of simulations on $30 \mathrm{~nm}$ tall dots were performed with out any crystalline anistropy, $K=0$. All of thesc simulations produced a hysteresis loop (that is $\left\langle M_{x}\right\rangle$ as a function of applied field) that were 'square'- the magnetic moment configuration switched abruptly at $H_{c}$.

Table $T$ is the summary of results for this series of simulations. The base case (Run 1) uses the experimentally determined size and the bulk value of $M_{s}$ for Co. Experimental results suggest that the effective $M_{s}$ may be as much as $80 \%$ lower in the dots and Run 2 uses this smaller value. We see that varying $M_{s}$ has little or essentially no affect on $H_{c}$ to within the resolution of the stepping of the applied field in these runs (20 Oe).

Runs 3 and 4 reduce size of the dots by $10 \%$ in each direction, but keeps the eccentricity $(a / b)$ the same. As expected, $H_{c}$ increases by $8 \%$ compared to the standard size. This is reasonable since the smaller dots have a larger demagnetization field and therefore take a larger external field to invoke the switching behavior. This is also confirmed by the fact that the magnetostatic energy for the smaller dots increased $\sim 20 \%$.

Finally, Runs 5 and 6 change the shape of the dots by reducing the eccentricity by $10 \%$ but keeps the volume of the dot the same. This small change of shape leads to the largest change of $H_{c}$ for this series of simulations with $K=0$. The reduction of $\sim 25 \%$ is not unexpected since more circular cross section allows for domain walls to form and propagate easier.

\section{Uniform Distributions of Crystalline Anistropy}

In this section, another series of simulations on $30 \mathrm{~nm}$ tall dots were performed. We fix the magnitude of the crystalline anistropy $K$ to $7.5 \times 10^{5} \mathrm{erg} / \mathrm{cm}^{3}$ but vary the orientation to be uniform along one of the Cartesian axis. 
Table II is the summary of results for this series of simulation. For $\hat{k}$ along the $x$ axis (Runs 712), we see that $H_{c}$ increases compared to when $K=0$. This unsurprising result is due to the crystalline anistropy 'freezing' the magnetic moments along the applied field direction, requiring an larger applied field to reverse the magnetic configuration. More interesting, reducing $M_{s}$ increases $H_{c}$. This is understandable since the maximum crystalline anisotropic field

$$
\mathbf{H}_{k}=\frac{2 K}{M_{s}^{2}}(\mathbf{M} \cdot \hat{k}) \hat{k}
$$

increases with decreasing $M_{s}$. Smaller dot size (Runs 9 and 10) or changing the shapc (Runs 11 and 12) scaled almost precisely as when $K=0$. Though, since the values of $H_{c}$ increased, changing the shape only decreased $H_{c}$ by $15 \%$ compared to the standard size.

When $\hat{k}$ is along the $z$-axis, it has minimal affect on $H_{c}$. It may decrease $H_{c}$ slightly for the larger $M_{s}$ runs. This weak response is not unexpected since the demaginzation field in the $z$ direction is very large due to the small thickness of the dots. Once the magnetic moments try to rotate out of the $x y$ plane, a very large opposing field is created in these thin structures.

However, when $\hat{k}$ is along the $y$-axis, more complicated behavior is seen. First, $H_{c}$ drops remarkably for all cases and, unlike the previous trends, the lower $M_{s}$ decreases $H_{c}$. Again, this is reasonable since now $\mathbf{H}_{k}$ is larger and reinforces the turning of the magnetic moments away from the applied field. Unlike the previous case, there is no resulting opposing field to stop the rotation. The exchange couple fields then forces the entire magnetic moment configuration to form a singe vortex. Changing the shape of the dot not only produces a $H_{c}=0$, but the onset of the single vortex actually occurs before the applied field switches signs (the numbers in '()' in Table II).

When $\hat{k}$ lies along the $x$ or $z$-axis, the resulting hysteresis loops are square as when $K=0$. However, when along the $y-a x i s, H_{c}$ is very small and all simulations report a formation of a single vortex in the magnetic configuration. This single vortex persists until a large enough external field ( 1300 Oe) when it's finally annihilated. Figure 1 shows a typical case.

\section{Random Distributions of Crystalline Anistropy}


In this section, we allow the crystalline anistropy distribution $\hat{k}$ to have random distributions. Unlike the previous results where the simulations were reproducible, multiple simulations with random $\hat{k}$ have to be done since results can vary dramatically from one random distribution to another. This fact is, perhaps, one of the most important conclusions of this section. Given the identical problem, including the probability function for generating the random $\hat{k}$ distributions, different distributions not only affect $H_{c}$ but also lead to either single or double vortex formation. (Of course, given the identical $\hat{k}$ distribution, the simulation returns the same results. That is, the algorithm itself is deterministic.)

Table III shows some results of different $\hat{k}$ distribution functions for the $30 \mathrm{~nm}$ thick dot. Identical entries mean only a different distribution was realized. All runs have $K=7.5 \times 10^{5}$ $\mathrm{erg} / \mathrm{cm}^{3}$.

' 3 -D' (Runs 25 and 26) means that $\hat{k}$ is isotropically random in $3-\mathrm{D}$ for each grain. As shown previously [2], higher $M_{s}$ values produce double vortex configuration and too low $H_{c}$ while lower $M_{s}$ tend to produce accurate $H_{c}$ predictions and about half single vortex patterns. '2-D' (Runs 27 and 28) means that $\hat{k}$ is random in the $x y$ plane and identically zero in $z$. Not surprisingly, the results do not change appreciably, except that $H_{c}$ increases slight. This is due to the fact that in 2-D it is more likely that any particular grain will have $\hat{k}$ along $x$ compared to a $3-\mathrm{D}$ distribution. We have seen that $\hat{k}$ along $x$ increases $H_{c}$. Halving the grain size (Run 29) didn't affect the $H_{c}$, but did tend to produce more single vortcx configuration at switching. Figure 1 shows the avcraged magnetic response for those simulations that produced single vortex states for both 2-D and 3-D configurations.

Reducing the size of the dots (Runs 30 and 31) resulted in higher $H_{c}$ for the lower $M_{s}$ values. Higher $M_{s}$ values still produced small $H_{c}$ compared to experiment. Changing the shape (Run 32) seem to suggest that even for the lower $M_{s}$ value, $H_{c}$ is underestimated by large factors.

The final run (33) tried to duplicate TEM pictures of the grain structure of the dots [10]. From those scans, the grains appear to grow mostly along the $z$-axis offset by a small random angle. It. was also seen that in the vast majority of the grains grew in pairs and it was suggested [10] that the 
$\hat{k}$ were coupled, or 'twinned', in these grains to be in opposite directions. Run 33 was produced by generating a random distribution of $\hat{k}$ in the $x y$ plane and a probability distribution for the polar angle, $\theta=\cos ^{-1} \mu$ given by:

$$
\frac{2\left(\mu-\mu_{l}\right)}{\left(1-\mu_{l}\right)^{2}}
$$

where $\mu_{l}=3\langle\mu\rangle-2$ and $\langle\mu\rangle$ is the average cosine of the polar angle in this distribution $\left(\theta=20^{\circ}\right.$ here). Integrating the above equation from $\mu_{l}$ to $\mu$ and setting the result to a uniformly distributed random number $[0-1]$ and solving for $\mu$ gives the required random $k_{z}$. Not only do the results agree well with experiment for $H_{c}$, but all runs produced a single vortex. A typical result (labeled "twinned") is shown in Figure 1.

Increasing the value of $K$ to the bulk value of Co were also performed. Unfortunately in most cases the results in $H_{c}$ did not come close to experimental results, typically much too large. Of those which did, typically very complex vortex behavior was seen. However, most of these runs did exhibit early and slow nucleation of the switching configuration.

\section{Conclusion}

In this paper, we have done numerical studies on polycrystalline Cobalt elliptical dots and their switching behavior. By simplify the real dots by first ignoring the crystalline anistropy and then using simple (uniform) distributions, insight has been gained on how the shape, size and magnetic moment magnitude affects the switching field and magnetic configuration.

Random distributions of the crystalline anistropy were then implemented. Different distributions (generated from the same probability function) generated different magnetic responses of the dots. Slow nucleation of the magnetic configuration were only realized with large values of the crystalline anistropy coefficient which rarely lead to the observed coercivity and magnetic configuration.

\section{Acknowledgments}

This work was performed under the auspices of the United States Department of Energy by Lawrence Livermore National Laboratory under Contract W-7405-ENG-48. 


\section{References}

1. W.F. Brown, "Micromagnetics", John Wiley \& Sons, New York, 1963.

2. G.J. Parker, C. Cerjan and D.W. Hewett, "Embedded curve boundary method for micromagnetic simulations", submitted to J. Magn. Magn. Mater.

3. A. Fernandez, M.R. Gibbons, M.A. Wall and C.J. Cerjan, J. Magn. Magn. Mater., 190, 71, (1998); A. Fernandez. and C.J. Cerjan, Appl. Phys. Lett. (to appear).

4. M.R. Gibbons, G.J. Parker, C.J. Cerjan and D.W. Hewett, "Finite difference micromagnetic simulation with self-consistent currents and smooth surfaces", J. Appl. Phys. (to appear). 
Table I: Shape, size and $M_{s}$ effects

\begin{tabular}{|c|c|c|c|c|}
\hline Run & $M_{s}\left(\mathrm{emu} / \mathrm{cm}^{3}\right)$ & $a(\mathrm{~nm})$ & $b(\mathrm{~nm})$ & $H_{c}(\mathrm{Oc})$ \\
\hline experiment & $1160-1450$ & 450 & 250 & 200 \\
1 & 1450 & 450 & 250 & 1250 \\
2 & 1160 & 450 & 250 & 1230 \\
3 & 1450 & 405 & 225 & 1350 \\
4 & 1160 & 405 & 225 & 1330 \\
5 & 1450 & 436.4 & 272.7 & 930 \\
6 & 1450 & 436.4 & 272.7 & 950 \\
\hline
\end{tabular}

Table II: Uniform crystalline anistropy effects

\begin{tabular}{|c|c|c|c|c|c|}
\hline Run & $\hat{k}$ & $M_{s}\left(\mathrm{emu} / \mathrm{cm}^{3}\right)$ & $a(\mathrm{~nm})$ & $b(\mathrm{~nm})$ & $H_{c}(\mathrm{Oe})$ \\
\hline 7 & $\hat{x}$ & 1450 & 450 & 250 & 2210 \\
8 & $\hat{x}$ & 1160 & 450 & 250 & 2430 \\
9 & $\hat{x}$ & 1450 & 405 & 225 & 2330 \\
10 & $\hat{x}$ & 1160 & 405 & 225 & 2530 \\
11 & $\hat{x}$ & 1450 & 436.4 & 272.7 & 1910 \\
12 & $\hat{x}$ & 1160 & 436.1 & 272.7 & 2150 \\
13 & $\hat{z}$ & 1450 & 450 & 250 & 1150 \\
14 & $\hat{z}$ & 1160 & 450 & 250 & 1210 \\
15 & $\hat{z}$ & 1450 & 405 & 225 & 1230 \\
16 & $\hat{z}$ & 1160 & 405 & 225 & 1310 \\
17 & $\hat{z}$ & 1450 & 436.4 & 272.7 & 910 \\
18 & $\hat{z}$ & 1160 & 436.4 & 272.7 & 950 \\
19 & $\hat{y}$ & 1450 & 450 & 250 & 150 \\
20 & $\hat{y}$ & 1160 & 450 & 250 & 10 \\
21 & $\hat{y}$ & 1450 & 405 & 225 & 230 \\
22 & $\hat{y}$ & 1160 & 405 & 225 & $0(50)$ \\
23 & $\hat{y}$ & 1450 & 436.4 & 272.7 & $0(370)$ \\
24 & $\hat{y}$ & 1160 & 436.4 & 272.7 & $0(370)$ \\
\hline
\end{tabular}


Table III: Random distrubtions of crystalline anistropy effects

\begin{tabular}{|c|c|c|c|c|c|c|}
\hline Run & $\hat{k}$ & $M_{s}\left(\mathrm{emu} / \mathrm{cm}^{3}\right)$ & $a(\mathrm{~nm})$ & $b(\mathrm{~nm})$ & $H_{c}(\mathrm{Oe})$ & Double or Singlc vortex \\
\hline Expt. & & & $\sim 450$ & $\sim 250$ & 200 & $\mathrm{~S}$ \\
\hline \multirow[t]{2}{*}{25} & $3-\mathrm{D}$ & 1450 & 450 & 250 & 20 & $\mathrm{D}$ \\
\hline & $3-\mathrm{D}$ & 1450 & 450 & 250 & 40 & $\mathrm{D}$ \\
\hline \multirow[t]{5}{*}{26} & $3-\mathrm{D}$ & 1160 & 450 & 250 & 254 & $\mathrm{D}$ \\
\hline & $3-\mathrm{D}$ & 1160 & 450 & 250 & 196 & S \\
\hline & $3-\mathrm{D}$ & 1160 & 450 & 250 & 215 & $\mathrm{~S}$ \\
\hline & $3-\mathrm{D}$ & 1160 & 450 & 250 & 195 & $\mathrm{D}$ \\
\hline & $3-\mathrm{D}$ & 1160 & 450 & 250 & 254 & $\mathrm{D}$ \\
\hline \multirow[t]{2}{*}{27} & $2-\mathrm{D}$ & 1450 & 450 & 250 & 26 & $\mathrm{~S}$ \\
\hline & $2-\mathrm{D}$ & 1450 & 450 & 250 & 58 & $\mathrm{D}$ \\
\hline \multirow[t]{4}{*}{28} & $2-\mathrm{D}$ & 1160 & 450 & 250 & 236 & $\mathrm{~S}$ \\
\hline & $2-\mathrm{D}$ & 1160 & 450 & 250 & 234 & $\mathrm{D}$ \\
\hline & $2-\mathrm{D}$ & 1160 & 450 & 250 & 235 & $\mathrm{D}$ \\
\hline & $2-\mathrm{D}$ & 1160 & 450 & 250 & 234 & $\mathrm{~S}$ \\
\hline \multirow[t]{5}{*}{29} & 3 -D, half grain & 1160 & 450 & 250 & 176 & $\mathrm{D}$ \\
\hline & 3-D, half grain & 1160 & 450 & 250 & 216 & $\mathrm{~S}$ \\
\hline & 3 -D, half grain & 1160 & 450 & 250 & 197 & S \\
\hline & $3-\mathrm{D}$, half grain & 1160 & 450 & 250 & 196 & S \\
\hline & $3-\mathrm{D}$, half grain & 1160 & 450 & 250 & 176 & $\mathrm{D}$ \\
\hline \multirow[t]{2}{*}{30} & 3-D & 1450 & 405 & 225 & 76 & $\mathrm{D}$ \\
\hline & $3-\mathrm{D}$ & 1450 & 405 & 225 & 17 & S \\
\hline \multirow[t]{2}{*}{31} & $3-\mathrm{D}$ & 1160 & 405 & 225 & 256 & $\mathrm{D}$ \\
\hline & $3-\mathrm{D}$ & 1160 & 405 & 225 & 293 & S \\
\hline \multirow[t]{2}{*}{32} & $3-\mathrm{D}$ & 1160 & 436.4 & 272.7 & 58 & $\mathrm{D}$ \\
\hline & 3-D & 1160 & 436.4 & 272.7 & 37 & $\mathrm{~S}$ \\
\hline \multirow[t]{2}{*}{33} & twinned & 1160 & 450 & 250 & 178 & S \\
\hline & twinned & 1160 & 450 & 250 & 187 & $\mathrm{~S}$ \\
\hline
\end{tabular}




\section{Figure Captions}

Fig. 1. Magnetic response of $30 \mathrm{~nm}$ thick Co dot: $a=450 \mathrm{~nm}, b=250 \mathrm{~nm}, M_{s}=1160 \mathrm{emu} / \mathrm{cm}^{3}$ and $K=7.5 \times 10^{5} \mathrm{erg} / \mathrm{cm}^{3}$. See text for details. 


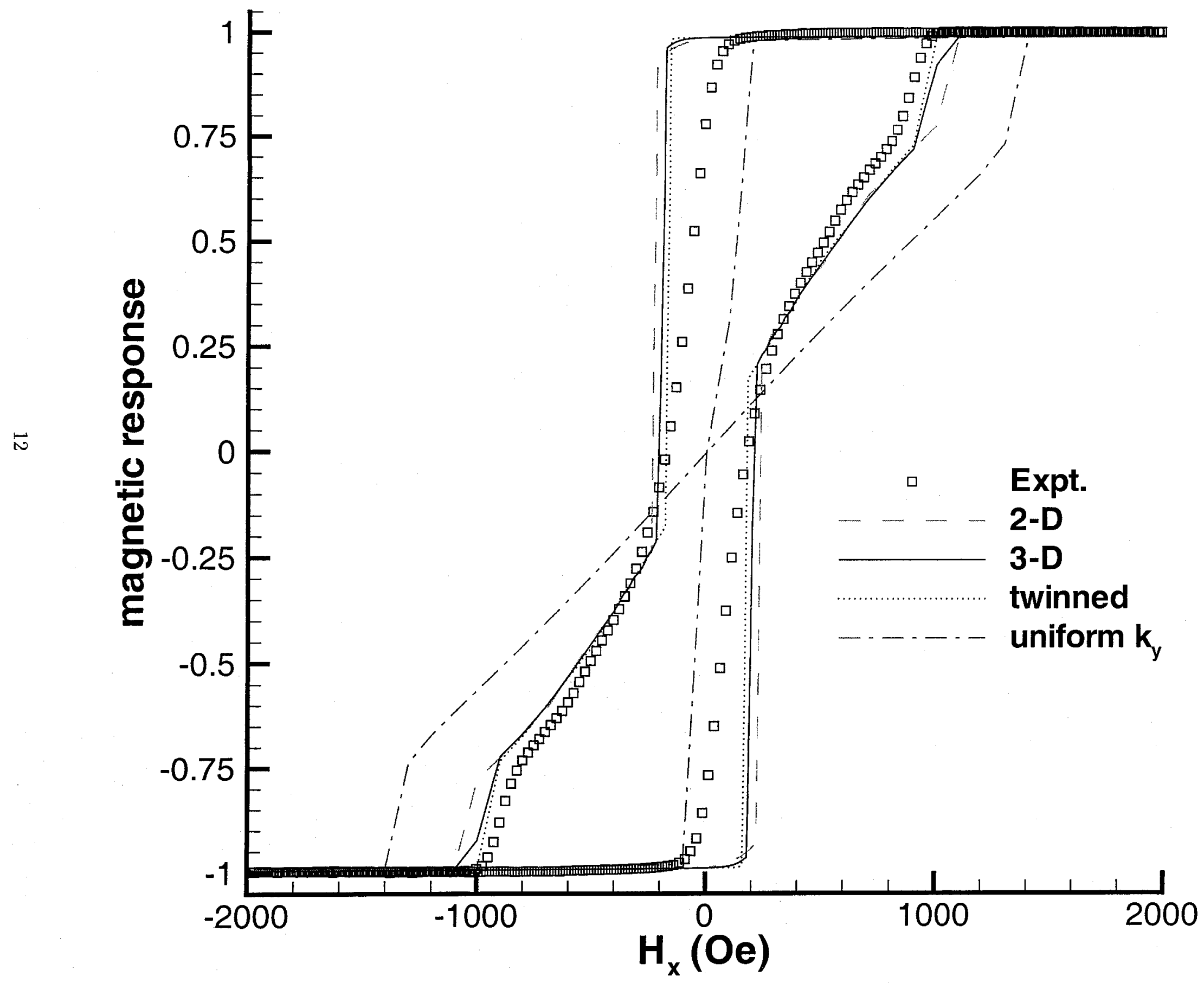

